Publication Types: Case report

\title{
Palatal perforations secondary to inhaled cocaine abuse. Presentation of five cases
}

P Serrano-Sánchez, JV Bagán ${ }^{2}$, Y Jiménez-Soriano ${ }^{3}$, G Sarrión ${ }^{1}$

${ }^{1}$ Dental surgeon. Postgraduate in Medicine and Oral Surgery. Faculty of Medicine and Dentistry, University of Valencia. Service of Stomatology, Valencia University General Hospital. Valencia, Spain

${ }^{2}$ DDS, PhD Chairman of Oral Medicine, Valencia University. Head of the Service of Stomatology. Valencia University General Hospital. Valencia, Spain

${ }^{3}$ DDS, PhD Professor of Oral Medicine. University of Valencia. Valencia. Spain

Correspondence:

Hospital General Universitario

Servicio de Estomatología

Avda. Tres Cruces s $/ n$

46014 - Valencia (Spain)

dr.serranosanchez@gmail.com

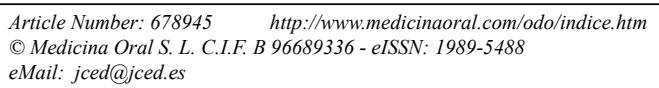

\begin{abstract}
The estimated incidence of complications associated with cocaine abuse is $4.8 \%$. Palatal perforation is a very infrequent condition within the range of complications associated with cocaine abuse. No epidemiological characteristics relating to patient age or gender have been established, and the diagnosis of the disorder requires a detailed clinical history, including antecedents of cocaine abuse. The clinical and radiological studies show palatal bone erosion and perforation of the soft or hard palate. The present study describes five cases of palatal perforation secondary to inhaled cocaine abuse.

As a result of the increase in cocaine use in recent years, particularly among young adults, clinicians must be alerted to the need to include this condition when establishing a differential diagnosis.
\end{abstract}

Key words: Cocaine, palatal perforation, inhalation, oronasal communication. 


\section{Introduction}

The estimated incidence of complications associated with cocaine abuse is $4.8 \%$. However, this figure is probably greater, since many cases go unnoticed, are not registered, or are not seen by the specialist. Cocaine-induced palatal perforation is a very infrequent complication. No epidemiological characteristics of this disorder relating to patient age or gender have been established $(1,2)$.

The diagnosis of this complication requires a detailed clinical history, including antecedents of cocaine abuse. Evaluation of the clinical and radiological studies is also required in order to identify palatal bone erosion and perforation of the soft or hard palate $(3,4)$.

The management approach is subject to controversy. The lack of will to abandon the habit, or the usual patient inefficacy in achieving complete rehabilitation, causes specialists to adopt a more conservative treatment approach, in many cases without proposing surgical sealing of the defect. The use of palatal obturators improves patient quality of life, avoiding socially embarrassing situations such as the passage of fluids and solids into the nasal passages and maxillary sinuses, or a nasal tone (5-7).

\section{Case Report}

The present study describes five cases of palatal perforation secondary to inhaled cocaine abuse diagnosed in the period between 2002-2008 in the Service of Stomatology of Valencia University General Hospital (Valencia, Spain)(Table 1).

The first case corresponds to a 32-year-old woman complaining of liquid passage from the oral cavity into the nasal cavities. Exploration revealed a painless oronasal lesion measuring $0.5 \mathrm{~cm}$ in greater diameter, located in the anterior portion of the hard palate, with no signs of inflammation or suppuration. Computed tomography revealed the absence of proprietary structures of the nasal cavities. This was the only case subjected to surgical treatment in the form of a pediculate flap, since the patient claimed to have stopped cocaine abuse. However, months later the lesion reappeared, and follow-up examination confirmed failure to abandon the habit.

The second case corresponded to a 29-year-old male with a painless lesion measuring $2 \mathrm{~cm}$ in diameter, located in the hard palate and affecting the midline (Fig. 1). The imaging studies likewise confirmed the lack of bone structure in the floor of the nasal cavities. Another consequence of inhaled cocaine abuse was the presence

\begin{tabular}{|l|l|l|l|l|l|l|l|}
\hline & $\begin{array}{c}\text { Age } \\
\text { (years) }\end{array}$ & \multicolumn{1}{|c|}{ Sex } & $\begin{array}{c}\text { Complications of } \\
\text { cocaine abuse }\end{array}$ & Pain & $\begin{array}{c}\text { No. oral } \\
\text { lesions }\end{array}$ & Lesion location & $\begin{array}{c}\text { Lesion } \\
\text { size }\end{array}$ \\
\hline$\# 1$ & 32 & Female & No & No & 1 & Hard palate & $0.5 \mathrm{~cm}$ \\
\hline$\# 2$ & 29 & Male & Pilonidal abscess & No & 1 & Hard palate & $2 \mathrm{~cm}$ \\
\hline$\# 3$ & 30 & Female & No & No & 1 & Hard palate & $2 \mathrm{~cm}$ \\
\hline$\# 4$ & 52 & Male & $\begin{array}{l}\text { Chronic pharyngi- } \\
\text { tis, palatal and para- } \\
\text { nasal necrosis }\end{array}$ & No & 1 & $\begin{array}{l}\text { Hard and soft } \\
\text { palate }\end{array}$ & $4 \mathrm{~cm}$ \\
\hline$\# 5$ & 50 & Female & Paranasal necrosis & No & 2 & $\begin{array}{l}\text { Hard and soft } \\
\text { palate }\end{array}$ & $\begin{array}{l}1 \mathrm{~cm}+ \\
3 \mathrm{~cm}\end{array}$ \\
\hline
\end{tabular}

Table 1. Characteristics of the five patients with palatal perforation secondary to inhaled cocaine abuse seen in the period between $2002-2008$ in the Service of Stomatology of Valencia University General Hospital (Valencia, Spain).
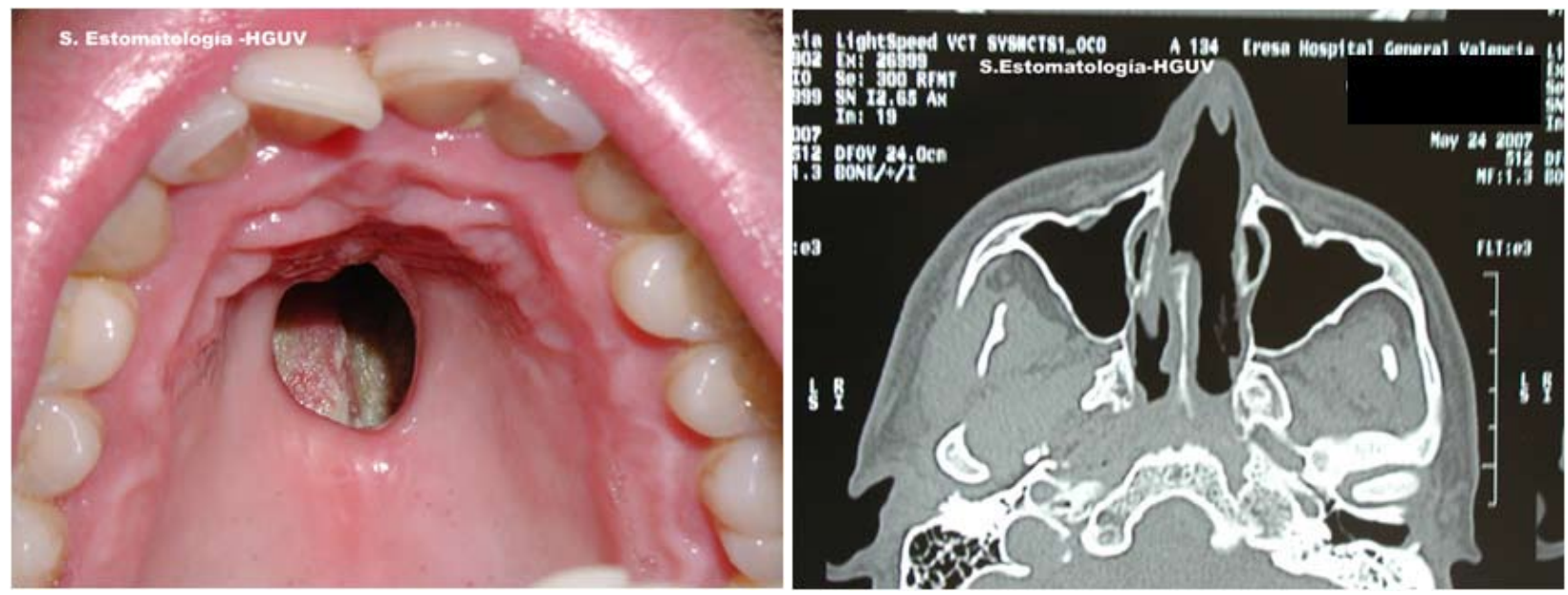

Fig. 1. Clinical and radiological view (axial computed tomography scan) of patient number 2. 

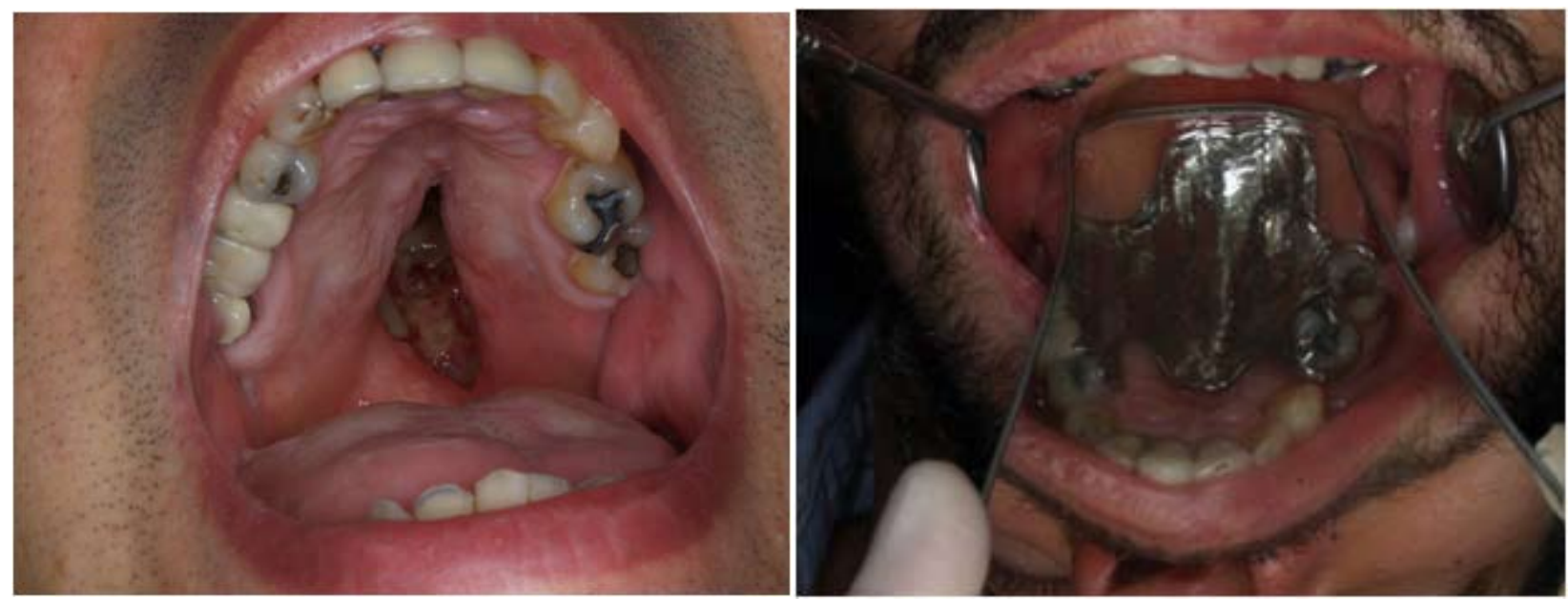

Fig. 2. Clinical views of patient number 4. Despite the extent of the lesion, fitting of the obturator completely sealed the defect.

of recurrent pilonidal abscesses as established from the medical records.

The third case corresponds to a 30-year-old woman with a smoking habit and regular alcohol consumption, presenting a perforation $1 \mathrm{~cm}$ in diameter in the hard palate close to its junction with the soft palate. In this case there were no other complications associated with inhaled cocaine abuse. Although intraoral examination showed the lesion to be smaller than in the previous case, the bone loss of the floor of the nasal cavities as evidenced by computed tomography was much greater.

The fourth case corresponded to a 52-year-old male of high social and economical status who presented a palatal perforation measuring $4 \mathrm{~cm}$ in diameter and affecting both the hard and soft palate (Fig. 2). Other complications associated with cocaine abuse were a hoarse nasal tone, the passage of liquids and food into the nasal cavities, with swallowing difficulties, heart disease, chronic pharyngitis and paranasal necrosis that was being treated by his ear, nose and throat specialist. Being aware of the cause of the lesion and of the difficulty of abandoning the habit, the patient requested treatment with a chromium and cobalt palatal obturator (this offering a lesser thickness than in the case of an acrylic resin obturator), which resulted in considerable improvement of his qua- lity of life.

The fifth case corresponds to a 50-year-old woman of very limited economical, social and cultural level. This patient reported for the removal of several teeth. The extraoral exploration revealed the lack of a nasal septum, deformity of the nares as a result of necrosis, and generalized edema of the entire middle third of the face. The intraoral exploration in turn revealed two independent lesions - one measuring $2 \mathrm{~cm}$ in diameter and located in the hard palate, and the other measuring $3 \mathrm{~cm}$ in size and located in the soft palate (Fig. 3). The perforations were asymptomatic, without suppuration or other general symptoms. She moreover also presented paranasal necrosis and chronic rhinitis, together with important swallowing and speech difficulties. The patient had been treated for her nasal necrosis time ago, but had failed to report for the programmed control visits. Computed tomography revealed the absence of nasal bone structures and erosion of the internal aspect of the right infraorbital zone in coronal image acquisition, with extension to the skull base in cross-sectional imaging.

\section{Discussion}

Cocaine-induced palatal perforation is a very infrequent complication. Cocaine is a potent vasoconstrictor with
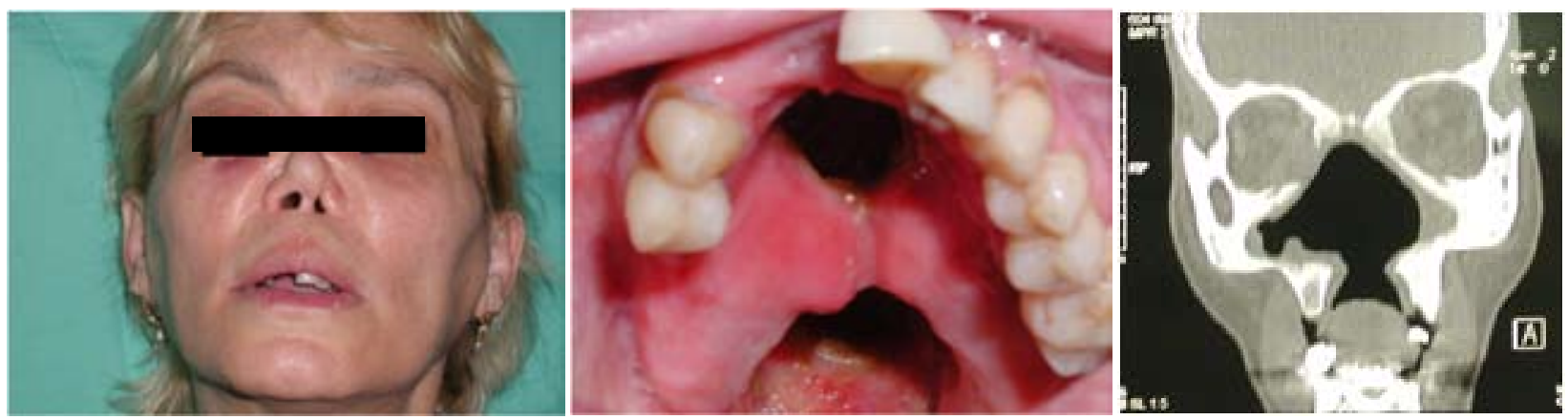

Fig. 3. Clinical views of patient number 5. Clinical extraoral and intraoral images and a coronal computed tomography scan. The latter shows the important bone destruction caused by continued cocaine abuse. 
psychoactive properties. The effects of the drug are mediated by its indirect sympathomimetic action, facilitating catecholaminergic neurotransmission with an increase in norepinephrine levels and lowered reuptake. Cocaine was introduced in medical practice in 1884 by Köller, due to its anesthetic action, since the drug blocks the sodium channels, interfering with nerve transmission $(8,9)$.

The drug is obtained from the coca leaf, and its production involves conversion of the leaves into a paste with the application of catalyzing agents such as sulfuric acid and gasoline, to yield cocaine hydrochloride (2).

There are different routes of administration in the context of substance abuse - the most common in the industrialized world being inhalation through the nose (snorting) $(2,10)$.

Cocaine consumption in Europe is on the rise. According to the annual report of the European Monitoring Center for Drugs and Drug Addiction, in the year 2007 a total of 4.5 million people consumed cocaine, versus 3.5 million the year before. The United Kingdom and Spain have the highest consumption rates, and $4-6 \%$ of all adolescents between 15-16 years of age have tried the drug (11).

The most important tissue effect is vasoconstriction. Ischemic necrosis and irritation are produced as a result of both the drug itself and of the adulterants commonly found in its composition. This, and the traumatic effect of the crystals inhaled at high speed, favor the appearance of recurrent nasal infections. This situation contributes to chronic tissue destruction $(7,12)$.

According to Jewers et al., another possible pathogenic mechanism is the presence of opiate receptors in macrophages and lymphocytes, causing immune alterations that would favor bacterial and fungal invasion (13).

The adverse effects of cocaine abuse include epistaxis, chronic rhinitis, anosmia, halitosis, perforation of the nasal septum and destruction of the lateral nasal walls. The associated neurological disorders in turn include cluster headache. On the other hand, individuals who frequently consume the drug tend to experience severe bruxism, with an increased incidence of joint and myofascial pain $(3,7,9)$.

The systemic complications of cocaine abuse in turn include arrhythmias, acute myocardial infarction, stroke, bronchospasm, pneumothorax, etc. $(2,8,13)$.

Oronasal palatine perforations are among the most extreme and rarely published side effects $(3,6)$.

The diagnosis of such lesions is based on the patient clinical history, including antecedents of sustained cocaine abuse. The differential diagnosis includes aggressive neoplasms, necrotizing inflammatory disorders such as atrophic rhinitis, tertiary syphilis, Wegener's granulomatosis (with positive antineutrophil cytoplasmic antibody (ANCA) serological findings), lupus erythemato- sus and lymphoproliferative conditions $(2,6-8,12,13)$. The treatment options comprise sealing of the defect, either surgically or with a removable obturator, once the lesion has been seen to remain stable. Such management also implies patient rehabilitation as regards cocaine abuse. The surgical technique is chosen according to the location and dimension of the lesion, the daily dose and duration of cocaine addiction, the presence of infections and the general patient conditions, among other factors. For reconstruction of the defect, the literature describes surgical techniques that are also used in application to cleft palates. In this context, local pediculate flaps, lingual grafts, temporal muscle flaps or oral adipose tissue grafting can be used. In the case of extensive lesions, a free microvascularized flap is indicated, with or without simultaneous bone transfer $(6,7)$.

The literature also describes the resolution of small or medium-sized defects using a Le Fort I osteotomy and bilateral adipose flap of the Bichat bulla, in those cases where a temporal or microvascularized flap is contraindicated (14).

The use of prosthetic obturators as a solution to situations that pose social problems for the patient is often indicated in cases characterized by palatal communications secondary to highly mutilating surgery. These obturators may be offered as an alternative for patients with palatal perforations caused by cocaine abuse who do not wish to undergo surgery, in those cases where the cost / benefit ratio is not favorable, in patients who cannot or do not wish to abandon the habit, or as a temporary measure before surgical treatment $(5,6)$.

Such obturators avoid nasal reflux, facilitating correct swallowing and sufficient speech performance. The only contraindication to such devices is patient tolerance of the obturator, since in some cases the obturator size required to fully seal the defect can cause nausea (5).

In conclusion, palatal perforation is an infrequent condition within the range of complications associated with cocaine abuse. Nevertheless, as a result of the increase in cocaine use in recent years, particularly among young adults, clinicians must be alerted to the need to include this condition when establishing a differential diagnosis.

\section{References}

1. Caravaca A, Casas F, Mochón A, De Luna A, San Martín A, Ruiz A. Centrofacial necrosis secondary to cocaine use. Acta Otorrinolaringol Esp. 1999;50: 414-6.

2. Padilla-Rosas M, Jimenez-Santos CI, García-González CL. Palatine perforation induced by cocaine. Med Oral Patol Oral Cir Bucal. 2006; 11:E239-42.

3. Peñarrocha M, Bagán JV, Peñarrocha MA, Silvestre FJ. Cluster headache and cocaine use. Oral Surg Oral Med Oral Pathol Oral Radiol Endod. 2000;90:271-4.

4. Di Cosola M, Turco M, Acero J, Navarro-Vila C, Cortelazzi R. Cocaine-related syndrome and palatal reconstruction: report of a series of cases. Int J Oral Maxillofac Surg. 2007;36:721-7.

5. Genden EM, Wallace DI, Okay D, Urken ML. Reconstruction of the 
hard palate using the radial forearm free flap: indications and outcomes. Head Neck. 2004;26: 808-14.

6. Goodger NM, Wang J, Pogrel MA. Palatal and nasal necrosis resulting from cocaine misuse. Br Dent J. 2005;198:333-4.

7. Di Cosola M, Turco M, Acero J, Navarro-Vila C, Cortelazzi R. Cocaine-related syndrome and palatal reconstruction: report of a series of cases. Int J Oral Maxillofac Surg. 2007;36:721-7.

8. Marí A, Arranz C, Gimeno X, Lluch J, Pericot J, Escuder O, et al. Nasal cocaine abuse and centrofacial destructive process: report of three cases including treatment. Oral Surg Oral Med Oral Pathol Oral Radiol Endod. 2002;93:435-9.

9. Gandara-Rey JM, Diniz-Freitas M, Gandara-Vila P, Blanco-Carrion A, Garcia-Garcia A. Lesions of the oral mucosa in cocaine users who apply the drug topically. Med Oral. 2002;7:103-7.

10. Tartaro G, Rauso R, Bux A, Santagata M, Colella G. An unusual oronasal fistula induced by prolonged cocaine snort. Case report and literature review. Minerva Stomatol. 2008;57: 203-10.

11. European Monitoring Centre for Drugs and Drugs Adiction [Internet]. Lisbon: 2007 Annual report: the state of the drugs problem in Europe [updated $2010 \mathrm{Feb}$ 10; cited $2010 \mathrm{Feb} 20$ ]. Available from: http://www.emcdda.europa.eu/.

12. Trimarchi M, Gregorini G, Facchetti F, Morassi ML, Manfredini $\mathrm{C}$, Maroldi R, et al. Cocaine-induced midline destructive lesions: clinical, radiographic, histopathologic, and serologic features and their differentiation from Wegener granulomatosis. Medicine (Baltimore). 2001;80:391-404.

13. Jewers WM, Rawal YB, Allen CM, Kalmar JR, Fox E, Chacon $\mathrm{GE}$, et al. Palatal perforation associated with intranasal prescription narcotic abuse. Oral Surg Oral Med Oral Pathol Oral Radiol Endod. 2005;99: 594-7.

14. Pelo S, Gasparini G, Di Petrillo A, Tassiello S, Longobardi G, Boniello R. Le Fort I osteotomy and the use of bilateral bichat bulla adipose flap: an effective new technique for reconstructing oronasal communications due to cocaine abuse. Ann Plast Surg. 2008;60: 49-52. 